\title{
Errata
}

\section{Estimation of Heat Flux through the Eastern Bering Strait}

Kohei Mizobata, Koji Shimada, Rebecca Woodgate, Sei-Ichi Saitoh and Jia Wang

Journal of Oceanography, Vol. 66, pp. 405-424, 2010

The authors list

"Kohei Mizobata, Koji Shimada, Rebecca Woodgate, Sei-ichi Saitoh and Jia Wang" should be changed as

"Kohei Mizobata, Koji Shimada, Sei-ichi Saitoh and Jia Wang". 